\title{
NEWSVENDOR PROBLEM WITH PRICING: PROPERTIES, ALGORITHMS, AND SIMULATION
}

\author{
Roger L. Zhan \\ ISE Department \\ University of Florida \\ Gainesville, FL 32611-6595, U.S.A.
}

\author{
Zuo-Jun Max Shen \\ IEOR Department \\ University of California \\ Berkeley, CA 94720-1777, U.S.A.
}

\begin{abstract}
When a newsvendor faces stochastic price-sensitive demands, he/she has to make the pricing and inventory decisions before the demand is realized. In the literature, this problem is typically solved by reducing it to an optimization problem over a single variable. In contrast, we treat this problem as a nonlinear system of two variables and provide some solution properties (e.g. existence, uniqueness) of the system. We also develop an iterative algorithm and a simulation based algorithm for this problem.
\end{abstract}

\section{INTRODUCTION}

The classic newsvendor problem is to make a single-period procurement decision of a single product under stochastic demands. This problem, because of its simple but elegant structure as well as its rich managerial insights, is a crucial building block of the stochastic inventory theory. It has been extensively studied over decades with extensions including different objectives and utility functions, multiple products with substitution, multiple locations, and different pricing strategies. For extensive reviews see Khouja (1999) and Porteus (1990).

In this paper, we study the extension that incorporates pricing decisions into the classic newsvendor problem. In this scenario, the newsvendor faces stochastic demands which are influenced by the price. The problem is to determine the procurement and pricing strategies that maximize the expected profit over a single period. We call this problem the Newsvendor Problem with Pricing (NPP).

The research on NPP started in 1950s with the work of Whitin (1955) and Mills (1959). Petruzzi and Dada (1999) provide a good review on this problem. Recent studies incorporate more market features such as price markdown and inventory sharing among multiple retailers (e.g., Sošić 2004 and Karakul and Chan 2004).

To the best of our knowledge, literature on NPP mostly focuses on transforming this problem of two decision variables (price and order quantity) to a problem of a single variable, either price $(p)$ or quantity $(Q)$, then solving the problem by using the first order conditions or an exhaustive search method. In this paper, we approach the problem by directly dealing with a system of two equations that are derived from the first order conditions. Using this approach, properties of the solutions to the problem, as well as a good geometric explanation of the problem, can be obtained. Furthermore, solution algorithms are developed based on this approach. In particular, we propose an efficient iterative algorithm and a simulation based algorithm. In the simulation based algorithm, random samples of demands are generated and the Sample Average Approximation (SAA) scheme and IPA (Infinitesimal Perturbation Analysis) gradients are used. The simulation based algorithm can be extended to more general cases, which involve multiple newsvendors.

The rest of the paper is organized as follows. Section 2 presents the model and the existence and uniqueness properties of the solution. Section 3 proposes the algorithms for the NPP problem. Finally, Section 4 summarizes the results and discusses future work.

\section{MODEL AND ITS PROPERTIES}

\subsection{Notation}

$$
\begin{aligned}
& p= \text { retail price. } \\
& Q= \text { order quantity. } \\
& v, s, c= \text { per unit salvage value, shortage cost, } \\
& \text { and purchase cost, respectively. } \\
& D(p, \epsilon)= a-b p+\epsilon . \\
& \epsilon= \text { stochastic term defined on the range } \\
& {[\text { A, B] with mean } \mu .} \\
& f(\cdot), F(\cdot)= \text { pdf and cdf of the distribution of } \epsilon . \\
& z= Q-(a-b p) . \\
& \Pi(z, p)= \text { expected profit function. } \\
& z^{*}, p^{*}=\quad \text { optimal solution. } \\
& p^{0}=\frac{a+b c+\mu}{2 b} .
\end{aligned}
$$




\section{Zhan and Shen}

\subsection{Model}

In this paper, the stochastic price-sensitive demand $D$ is modeled in an additive demand form, i.e., $D(p, \epsilon)=a-$ $b p+\epsilon(a>0, b>0) . \epsilon$ is a random variable defined on [A, B] with mean $\mu$, cumulative distribution function (cdf) $F(\cdot)$ and probability density function (pdf) $f(\cdot)$. Before the stochastic term $\epsilon$ is realized, the newsvendor determines simultaneously an order quantity, $Q$, and a retail price, $p$, to maximize the expected profit. We also assume that $v<c$ to avoid the trivial solution.

Denote $x^{+}=\max \{x, 0\}$, the newsvendor's profit can be expressed as the difference between the revenue and the total cost:

$$
\begin{aligned}
\pi(Q, p)= & p \min \{Q, D(p, \epsilon)\}-c Q+v(Q-D(p, \epsilon))^{+} \\
& -s(D(p, \epsilon)-Q)^{+} .
\end{aligned}
$$

This profit function can be rewritten by substituting $D(p, \epsilon)$ with $a-b p+\epsilon$ and defining $z=Q-(a-b p)$ :

$$
\begin{aligned}
\pi(z, p)= & p(a-b p+\min \{z, \epsilon\})-c(a-b p+z) \\
& +v(z-\epsilon)^{+}-s(\epsilon-z)^{+} .
\end{aligned}
$$

This variable transformation simplifies the computations and its economic meaning is provided in Petruzzi and Dada (1999). Noticing that $(x-y)^{+}=x-\min \{x, y\}$, the profit function can be further expressed as:

$$
\begin{aligned}
\pi(z, p)= & (p-c)(a-b p)-(c-v) z-s \epsilon \\
& +(p+s-v) \min \{z, \epsilon\},
\end{aligned}
$$

with the expected value

$$
\begin{aligned}
\Pi(z, p)= & E[\pi(z, p)]=(p-c)(a-b p)-(c-v) z \\
& -s \mu+(p+s-v) E(\min \{z, \epsilon\}) .
\end{aligned}
$$

The objective of the newsvendor is to maximize the expected profit $\Pi(z, p)$ by choosing $z$ and $p$. However, the optimal solution is not necessarily an interior solution, in particular, the value of $z$ can be on the boundary, i.e., $A$ or $B$. In the next subsection, we explore the conditions that guarantee the existence of the interior optimal solution. The geometric explanation of these condition is also provided.

\subsection{Solution Properties}

Define $\Theta(z)=\int_{z}^{B}(u-z) f(u) d u$. Notice that

$E(\min \{z, \epsilon\})=\int_{A}^{z} u f(u) d u+\int_{z}^{B} z f(u) d u=\mu-\Theta(z)$, and

$$
\frac{\partial(E(\min \{z, \epsilon\}))}{\partial z}=z f(z)+\frac{\partial(z(1-F(z)))}{\partial z}=1-F(z) .
$$

The first partial derivatives of $\Pi(z, p)$ are

$$
\begin{aligned}
\frac{\partial \Pi(z, p)}{\partial z} & =-(c-v)+(p+s-v)[1-F(z)], \\
\frac{\partial \Pi(z, p)}{\partial p} & =a+b c+\mu-2 b p-\Theta(z) \\
& =2 b\left(p^{0}-p\right)-\Theta(z) .
\end{aligned}
$$

Equations (5) and (6) imply the relationship between $z$ and $p$ as following:

$$
\begin{aligned}
z & \equiv z(p)=F^{-1}\left(\frac{p+s-c}{p+s-v}\right), \\
p & \equiv p(z)=p^{0}-\frac{\Theta(z)}{2 b} .
\end{aligned}
$$

Equation (7) is simply the solution formulation for the classic newsvendor problem. It naturally requires that $p \geq c-s$. And Equation (8) implies that $p \leq p^{0}$. Thus, the boundary condition for $p$ is $c-s \leq p \leq p^{0}$.

In the literature, researchers usually utilize Equation (8) to reduce the original problem to an optimization problem over a single variable $z$. Our approach, however, works on these two first order conditions directly, and provides more insights into the problem.

Let $r(z)=\frac{f(z)}{1-F(z)}$, we have

$$
\begin{aligned}
\frac{\partial z(p)}{\partial p} & =\frac{1}{r(z)(p+s-v)}, \\
\frac{\partial p(z)}{\partial z} & =\frac{1-F(z)}{2 b} .
\end{aligned}
$$

Equations (9) and (10) imply that the functions $z(p)$ and $p(z)$ increase in $p$ and $z$, respectively.

And the second partial derivatives are

$$
\begin{aligned}
\frac{\partial^{2} z(p)}{\partial p^{2}} & =-\frac{\frac{d r(z)}{d z} \frac{1}{r(z)}+r(z)}{(p+s-v)^{2} r^{2}(z)} \\
\frac{\partial^{2} p(z)}{\partial z^{2}} & =-\frac{f(z)}{2 b}<0 .
\end{aligned}
$$

Thus function $p(z)$ is concave in $z$ and based on Equation (11) the following lemma is established.

Lemma 1 If $F(\cdot)$ is a distribution function satisfying the condition $r^{2}(z)+\frac{d r(z)}{d z}>0$ for $z \in[A, B], z(p)$ is concave in $p$.

This condition is satisfied by all nondecreasing hazard rate distributions, which include $P F_{2}$ distributions and the 
log-normal distribution (Barlow and Proschan 1975). In particular, uniform, normal, logistic, chi-squared and exponential distributions all belong to this category (Bagnoli and Bergstrom 1989).

The next result follows from Lemma 1 and the fact that if functions $z(p)$ and $p(z)$ are increasing and concave in $p$ and $z$, respectively, then the system of the equations $z=z(p)$ and $p=p(z)$ has at most two solutions.

Lemma 2 If the condition for Lemma 1 is satisfied, then the system of Equations (7) and (8) has at most two solutions.

Geometrically, the lemma states that the two curves in Figure 1 have at most two intersections. We denote the upper intersection as "UC" and the lower intersection as "LC".

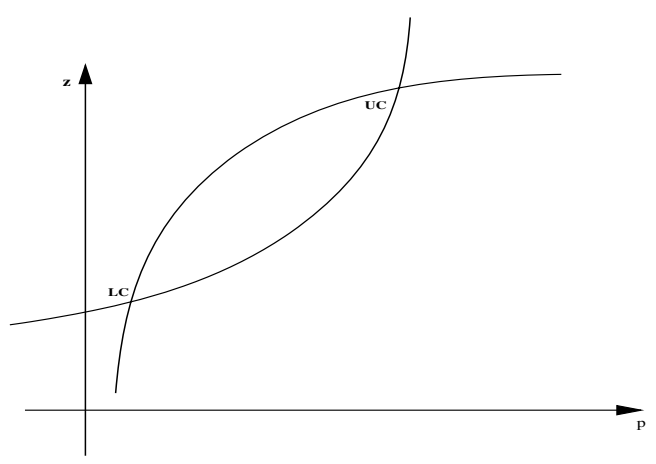

Figure 1: Two Solutions

By considering the boundary conditions, $A \leq z \leq B$ and $c-s \leq p \leq p^{0}$, properties of the NPP problem can be established.

Proposition 1 If the condition for Lemma 1 is satisfied and $a-b(c-2 s)+A>0$, then equations (7) and (8) have a unique solution, which is also optimal for the problem.

The condition $a-b(c-2 s)+A>0$, i.e., $\frac{a+b c+A}{2 b}>c-s$ is to ensure there is only one intersection of the two curves in the feasible region. This case is geometrically shown in Figure 2.

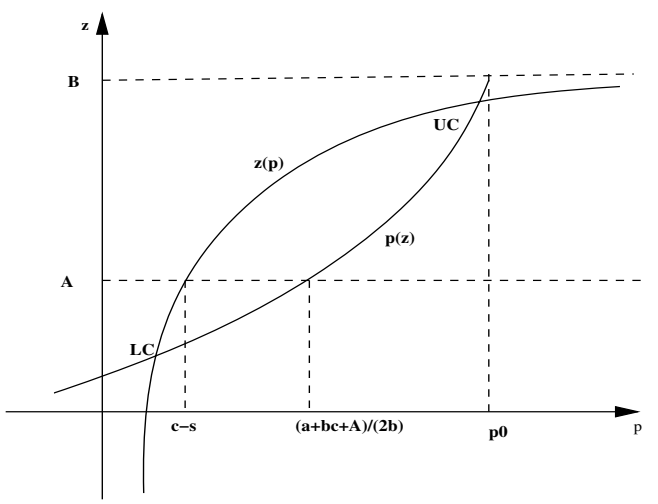

Figure 2: Unique Solution

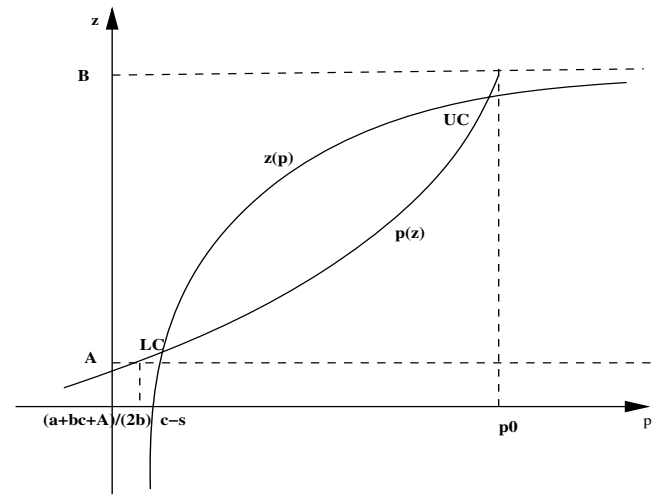

Figure 3: Two Solutions

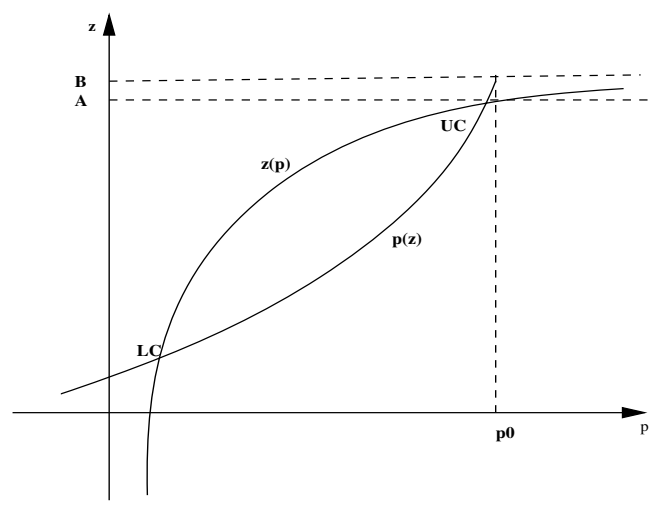

Figure 4: No Solution

If the condition $a-b(c-2 s)+A>0$ is not satisfied, then there are two scenarios. The first scenario is where Line A in Figure 2 moves lower than point "LC", and the system has two solutions (see Figure 3); the other scenario is where Line A moves higher than point "UC", and the system then has no solution, which implies NPP problem has no interior solution (see Figure 4). The following propositions characterize these two cases.

Proposition 2 If the condition for Lemma 1 is satisfied, $a-b(c-2 s)+A<0$ and $f(A)<\frac{1}{a+b(c+2 s-2 v)+A}$, then equations (7) and (8) have two solutions. The one with the larger $p$ value is the optimal solution for the NPP problem.

Proposition 3 If the condition for Lemma 1 is satisfied, $a-b(c-2 s)+A<0$ and $f(A)>\frac{1}{a+b(c+2 s-2 v)+A}$, then equations (7) and (8) have no solution. The NPP problem has the optimal solution on the boundary.

All the above properties are derived under the condition that Lemma 1 holds. If Lemma 1 does not hold, an exhaustive search method is not avoidable. In the next section, we develop the algorithms that are based on the properties derived in this section. 


\section{Zhan and Shen}

\section{ALGORITHMS}

Propositions 1 and 2 state the conditions for the NPP problem to have an interior solution. Past research usually limited the analysis on theoretical results, identified some simple cases that can be solved analytically and did not present algorithms that could be used to solve more general problems. In contrast, we develop an iterative algorithm, as well as a simulation based algorithm, to solve the system of Equations (7) and (8), which leads to the solution for the NPP problem under the conditions given by Propositions 1 and 2 .

\subsection{Iterative Algorithm}

Recall the relationship between $z$ and $p$ :

$$
\begin{aligned}
& z \equiv z(p)=F^{-1}\left(\frac{p+s-c}{p+s-v}\right) \\
& p \equiv p(z)=p^{0}-\frac{\Theta(z)}{2 b} .
\end{aligned}
$$

As shown later in Proposition 4 of this section, the above system can be solved iteratively and the solution converges to the optimal one. The algorithm works in this fashion: Start with an initial price, $p^{0}$, the value of $z$ can be calculated using Equation (7), and with Equation (8), a new price can be uniquely determined. This new price can then be used to update $z$ in the next iteration. The algorithm repeats this process till the optimal solution is found.

The procedure is summarized in a pseudo-code format in the following, where $\delta$ represents the precision that is used in the stopping criterion. In practice, we can also simply specify the number of the iterations as the stopping criterion.

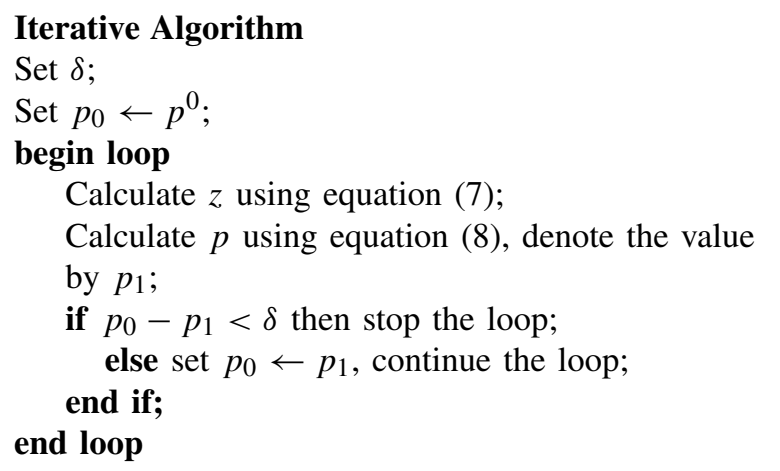

If the initial price starts from $p^{0}$, the above algorithm finds the solution to the system of Equations (7) and (8), which has a larger value of $p$ if the system has two solutions as shown in Proposition 2. The following proposition shows that the algorithm converges to the optimal solution, $z^{*}$ and $p^{*}$.

Proposition 4 Starting from $p^{0}$, the iterative algorithm converges to the optimal solution, $z^{*}$ and $p^{*}$.
Proof. From Equation (8) and $\Theta(z) \geq 0$, we know that $p^{0} \geq p^{*}$. To prove the convergence result, we examine the two sequences: $p_{0}=p^{0}, p_{1}=p\left(z_{0}\right), p_{2}=p\left(z_{1}\right) \ldots$ and $z_{0}=z\left(p_{0}\right), z_{1}=z\left(p_{1}\right), z_{2}=z\left(p_{2}\right) \ldots$, where $p(\cdot)$ and $z(\cdot)$ are the functions given in Equations (7) and (8).

Since $p_{0}=p^{0} \geq p^{*}$, due to the monotonicity of the function $z(\cdot), z_{0} \geq z^{*}$. Similarly, due to the monotonicity of the function $p(\cdot)$, we get $p_{0} \geq p_{1} \geq p^{*}$. The same analysis carries on while the algorithm keeps producing the elements in the sequences. That is, we have $p_{0} \geq p_{1} \geq p_{2} \ldots \geq p^{*}$ and $z_{0} \geq z_{1} \geq z_{2} \ldots \geq z^{*}$. Therefor these two sequence are monotonically decreasing and bounded. Thus they will converge to $p^{*}$ and $z^{*}$ respectively.

\subsection{Simulation Based Algorithm}

The iterative algorithm will work well and converge to the optimal solution very fast, if it is not difficult to solve Equation (7) analytically and easy to compute $\Theta(z)$ in Equation (8). To bypass these two potential difficulties, we need to resort to a different approach. Therefore, parallel to the iterative algorithm, we propose a simulation based algorithm to solve the NPP problem, which will work particularly well if the random instance can be easily generated. The simulation based algorithm is more promising if we want to extend the algorithm to deal with more complicated cases which may involve multiple newsvendors.

From Equation (4), the first order conditions can be written as

$$
\begin{aligned}
\frac{\partial \Pi(z, p)}{\partial z}= & -(c-v) \\
& +(p+s-v) \frac{\partial E(\min (\epsilon, z))}{\partial z}, \\
\frac{\partial \Pi(z, p)}{\partial p}= & -2 b p+b c+a+E(\min (\epsilon, z)) .
\end{aligned}
$$

The information of Equation (13) can be used to update the value of $z$ by the gradient search method. However, we need to estimate $\frac{\partial E(\min (\epsilon, z))}{\partial z}$ by $E\left(\frac{\partial(\min (\epsilon, z))}{\partial z}\right)$. This estimation is justified by the unbiased IPA estimator for the gradient because $\min (\epsilon, z)$ is almost surely continuous with respect to $z$. A detailed discussion of the application of IPA can be found in $\mathrm{Fu}$ (1994). Furthermore, the expectation is approximated by the sample average value, which is usually called the Sample Average Approximation (SAA) scheme.

The procedure is summarized in a pseudo-code format in the following, where $U$ represents the number of regenerative cycles, and $a_{k}$ represents the step size at iteration $k$, and $\delta$ represents the precision that is used in the stopping criterion. In practice, we can use other stopping criteria such as a preset number of iterations. 


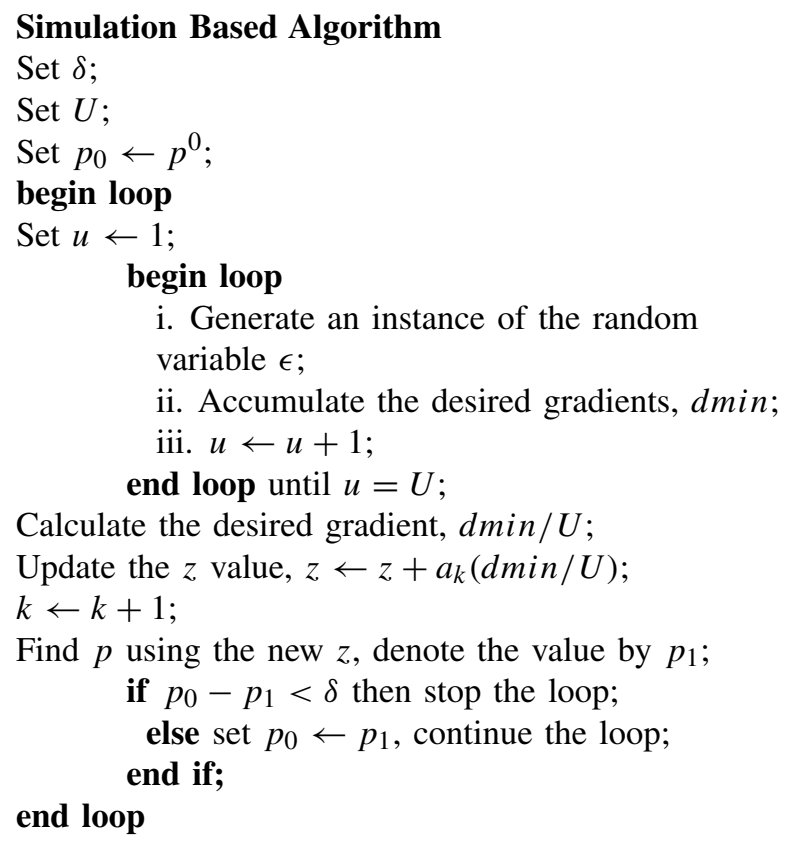

In step (ii) of the algorithm, we use IPA to compute the gradient. In the computer implementation, it works as follows: if the instance of $\epsilon$ is greater than $z$, then $d \min =d \min +p+s-c$; otherwise $d \min =d \min -c+v$. These equalities are derived from Equation (13). Starting with $d \min =0$ and dividing $d \min$ by $U$ yield the derivative estimation.

\subsection{Numerical Results}

In this section, we use two small numerical examples to illustrate the quality of the solutions from the the simulation based algorithm.

Example 1 In this example, the parameters are set as $v=0.5, s=c=1, a=200, b=35$, and $\epsilon$ follows the normal distribution with mean of zero and standard deviation of 20. Starting from $p^{0}=3.357$, the iterative algorithm gives the optimal solution $p^{*}=3.3385$ and $z^{*}=22.5033$.

We set $U=100$ in the simulation based algorithm. The program is coded in Matlab and results from 10 runs are reported in Table 1.

Example 2 In this example, the parameters are set as $v=0.5, s=c=1, a=200, b=35$, and $\epsilon$ is exponentially distributed with mean value of 10 . The iterative algorithm gives the optimal solution $p^{*}=3.4821$ and $z^{*}=20.7495$. The simulation solutions are shown in Table 2 .

Tables 1 and 2 clearly indicate that we can find the high quality solutions that are very close to the optimal solutions using the simulation based algorithm.

Although this paper mainly considers the single newsvendor problem, we believe the simulation based algorithm would have more advantage over the analytical algorithms in scenarios with multiple newsvendors.
Table 1: Simulation Algorithm: Normal Distribution

\begin{tabular}{|c|c|c|}
\hline Run No. & $p$ & $z$ \\
\hline 1 & 3.3285 & 22.4839 \\
2 & 3.3396 & 22.5082 \\
3 & 3.3431 & 22.4387 \\
4 & 3.3378 & 22.5185 \\
5 & 3.3365 & 22.5516 \\
6 & 3.3379 & 22.5288 \\
7 & 3.3320 & 22.4193 \\
8 & 3.3415 & 22.5225 \\
9 & 3.3407 & 22.4822 \\
10 & 3.3380 & 22.4869 \\
\hline Mean & 3.3376 & 22.4941 \\
Std. Dev & 0.0044 & 0.0409 \\
\hline Optimal & 3.3385 & 22.5033 \\
\hline
\end{tabular}

Table 2: Simulation Algorithm: Exponential Distribution

\begin{tabular}{|c|c|c|}
\hline Run No. & $p$ & $z$ \\
\hline 1 & 3.4754 & 20.7815 \\
2 & 3.4838 & 21.0357 \\
3 & 3.4879 & 20.7068 \\
4 & 3.4764 & 20.8121 \\
5 & 3.3786 & 20.6466 \\
6 & 3.3758 & 20.5008 \\
7 & 3.4870 & 20.6694 \\
8 & 3.4812 & 20.7523 \\
9 & 3.4854 & 20.8333 \\
10 & 3.4802 & 20.6616 \\
\hline Mean & 3.4812 & 20.7400 \\
Std. Dev & 0.0047 & 0.1420 \\
\hline Optimal & 3.4821 & 20.7495 \\
\hline
\end{tabular}

\section{CONCLUSIONS}

In this paper, we analyze, from a different perspective, the solution properties of the newsvendor problem with pricing. The analysis focuses on the system of the two equations derived from the first order conditions. The derived properties guide us to develop both the iterative algorithm and the simulation based algorithms.

There are several extensions to this paper. The simulation based algorithm can be generalized to deal with more complicated scenarios which may involve multiple products and multiple newsvendors. The model analyzed in this paper uses the additive demand form. Extending the model to other demand forms, such as multiplicative demand form, is also an interesting topic for future research. 


\section{Zhan and Shen}

\section{REFERENCES}

Bagnoli, M., and T. Bergstrom. 1989. Log-concave probability and its applications. University of Michigan. Working Paper. Available at <http://www. econ. ucsb.edu/ tedb/Theory/delta.pdf $>$.

Barlow, R. E., and F. Proschan. 1975. Statistical theory of reliability and life testing: Probability models. New York: Holt, Rinehart, and Winston.

Fu, M. C. 1994. Optimization via simulation: A review. Annals of Operations Research 53:199-248.

Karakul, M., and L. M. A. Chan. 2004. Newsvendor problem of a monopolist with clearance markets. YAEM 2004. Available at <http://yaem2004. cukurova.edu.tr/>.

Khouja, M. 1999. The single-period (news-vendor) problem: literature review and suggestions for future research. Omega, the International Journal of Management Science 27:537-553.

Mills, E. S. 1959. Uncertainty and price theory. Quarterly Journal of Economics 73:116-130.

Petruzzi, N. C., and M. Dada. 1999. Pricing and the newsvendor problem: a review with extensions. Operations Research 47(2):183-194.

Porteus, E. L. 1990. Stochastic inventory theory. In Handooks in $O R \& M S$, ed. D. P. Heyman and M. J. Sobel, 605-652. Elsevier, North-Holland.

Sošić, G. 2004. Price-setting newsvendors: Effects of price markdowns and inventory sharing. University of Southern California. Working paper. Available at <http://www-rcf.usc.edu/ sosic/ pricesetting.pdf $>$.

Whitin, T. M. 1955. Inventory control and price theory. Management Science 2:61-68.

\section{AUTHOR BIOGRAPHIES}

ROGER LEZHOU ZHAN is a Ph.D. candidate in the Department of Industrial and Systems Engineering at the University of Florida. His research interests include auction design, decisions under uncertainty and global optimization. $\mathrm{He}$ is a member of IIE, INFORMS, and SIAM. His e-mail address is <zhan@ufl. edu>.

ZUO-JUN MAX SHEN is an assistant professor in the Department of Industrial Engineering and Operations Research at University of California-Berkeley. $\mathrm{He}$ obtained his Ph.D. in Industrial Engineering and Management Sciences from Northwestern University. Before joining Berkeley, he taught in the Department of Industrial and Systems Engineering at the University of Florida. Dr. Shen's primary research interests are in the general area of integrated supply chain design and management, and practical mechanism design. His e-mail address is <shendieor.berkeley.edu>, and his web page is <http://www. ieor. berkeley. edu/ shen/>. 